Pensamiento Crítico Vol. 18 № 1, pp. 105-122

\title{
Eva y crisis financiera mundial
}

\author{
Dr. Raimundo Renaun Pacheco Mexzon
}

\section{RESUMEN}

A partir del año 2,008 en que se inicia en Estados Unidos de Norte América la crisis financiera, a la fecha, Junio del año 2,013 ha tomado dimensiones preocupantes a nivel mundial afectando no sólo el aspecto económico sino también el aspecto social y empresarial. En este marco nos preguntamos qué pasaría con la creación de valor económico de una empresa de mantenerse esta situación. A nuestro entender la empresa se vería afectada pudiendo destruir en vez de crear valor económico.

Palabras claves: Crisis Financiera Mundial. EVA (Economic Value Added)

\section{ABSTRACT}

From the year 2,008 which starts in the United States of America financial crisis, to date, 2,013 year June has taken alarming proportions worldwide affecting not only the economic but also the social and business. In this framework we wonder what would happen to economic value creation of a company to keep this situation. To our knowledge the company would be affected can destroy rather than create economic value.

Keywords: Global Financial Crisis. EVA (Economic Value Added) 


\section{Pensamiento Crítico Vol. 18. No I}

\section{Los orígenes de la crisis mundial}

Los estudios de investigación respecto a los orígenes de la crisis económica y financiera mundial establecen que los factores determinantes de la actual crisis son de naturaleza estructural.

Para la CEPAL (Comisión Económica para América Latina y el Caribe) por ejemplo estos factores son:

\section{En primer término:}

- La inadecuada regulación o carencia de cualquier tipo de regulación de las instituciones $y$

- Prácticas muy riesgosas de la denominada nueva arquitectura financiera internacional, compuesta por un sistema global de grandes bancos de inversión, fondos de cobertura y vehículos especiales de inversión.

\section{En segundo lugar:}

- Los fuertes desequilibrios mundiales que se registran: entre el trabajo y la circulación de capitales.

- El déficit comercial de los Estados Unidos y

- El predominio del capital financiero sobre el capital productivo.

Según un estudio de la CEPAL "la burbuja del mercado de crédito hipotecario de alto riesgo en Estados Unidos y la consiguiente deflación de la deuda, junto con el largo período de abundante liquidez y las bajas tasas de interés antes de la crisis, llevaron a los inversionistas a buscar mayor rentabilidad y a subestimar los riesgos. Por otro lado el alto nivel de endeudamiento del sector privado, sobre todo de los hogares estadounidenses, reflejan los factores estructurales de esta crisis".

No perder de vista que en los Estados Unidos, la crisis estuvo determinada por incentivos perversos y riesgos excesivos en el "sistema financiero sombra", que incluye a intermediarios financieros escasamente regulados e instrumentos de seguros complejos y poco claros que no se valoraron correctamente. 


\section{Raimundo Renaun Pacheco Mexzon}

\section{Consecuencias y contagios de la crisis a nivel mundial}

El estudio de la CEPAL nos indica que el alto grado de apalancamiento y el amplio riesgo sistémico generados con este sistema traspasaron la frontera estadounidense y provocaron tensiones que no se habían observado en las últimas tres cuartas partes del siglo.

Este riesgo sistémico se fue incrementando de manera progresiva en el sistema y su ignorancia trajo la conmoción en el mercado de las hipotecas de alto riesgo y la pérdida de valor de una enorme cantidad de derivados construidos sobre la base de dichas hipotecas. De esta manera las condiciones económicas mundiales sufrieron un marcado deterioro desde mediados de septiembre de 2008.

Este deterioro comenzó a mediados de 2007, cuando el aumento de la tasa de desempleo de los Estados Unidos provocó el colapso del mercado de las hipotecas de alto riesgo. La crisis de este mercado comenzó a repercutir rápidamente en los estados financieros de instituciones financieras sólidas, que tenían y vendían derivados con hipotecas de alto riesgo como activos subyacentes.

Al mismo tiempo, las fuertes alzas de los precios de los alimentos y la energía golpeaban a la economía mundial, lo que llevó a algunos bancos centrales a aumentar las tasas de interés para controlar las presiones inflacionarias, mientras otros simplemente mantenían las estrictas condiciones monetarias adoptadas en la etapa de prosperidad.

La CEPAL predijo que la gran volatilidad de los precios de las materias primas, sobre todo alimentos e hidrocarburos, era un fenómeno global atribuible a una combinación de factores. Algunos de ellos son de índole estructural como: el aumento de la población y de los ingresos, la creciente demanda de alimentos; la rápida industrialización de Asia, que promovió la demanda de materia prima y energía, y el otorgamiento generalizado de subsidios a la producción de biocombustibles en Europa y los Estados Unidos.

Hay otros factores de naturaleza más especulativa, como el exceso de liquidez y la influencia de los fondos de cobertura, que cada vez se orientan más hacia los mercados de los productos básicos. 


\section{Pensamiento Crítico Vol. I8. Nº}

Todo esto nos permite confirmar que hay tres fenómenos interrelacionados que se refuerzan mutuamente y que hoy sacuden a la economía mundial:

- La crisis financiera.

- La desaceleración del crecimiento y

- Los cambios de los precios relativos internacionales.

\section{Derrumbe de los mercados financieros}

La quiebra de Lehman Brothers marcó el comienzo del derrumbe de los mercados financieros mundiales, al evaporarse la confianza entre las instituciones financieras. Tras el colapso de esa entidad, los mercados monetarios mundiales a corto plazo se paralizaron y las transacciones comerciales a corto plazo se volvieron sumamente onerosas.

El mercado de títulos comerciales a corto plazo se paralizó y nadie estaba dispuesto a prestar. Desde entonces, los diferenciales de crédito se ampliaron significativamente, las bolsas se desplomaron y las economías del mundo tambalean.

El contagio financiero se extendió al resto de la economía y al mundo en general. La respuesta a la crisis se centró en desbloquear el crédito y evitar mayores daños a la economía mundial, pero la crisis ya golpea también a la región de América Latina y el Caribe y reina la incertidumbre.

Si bien los países del G-20 acordaron un conjunto de principios para transformar los mercados financieros, la reforma del sistema regulatorio mundial aún está a la esperar a que se aclare el panorama en cuanto a lo que se ha aprendido de esta crisis y hasta qué punto la regulación debe ser transnacional.

Pese a que esta vez los mercados emergentes no fueron los que provocaron la crisis, hoy su demanda agregada es más vulnerable a una contracción de la actividad económica mundial debido a la dependencia de la demanda externa y la inversión extranjera. Las instituciones financieras y los fondos de cobertura de las economías desarrolladas rápidamente retiraron grandes sumas de dinero de los mercados 


\section{Raimundo Renaun Pacheco Mexzon}

emergentes, lo que causó problemas a los bancos y mercados locales. Las líneas de crédito, fundamentales para las transacciones internacionales, también se congelaron, lo que afectó el comercio y redujo los ingresos de exportaciones.

Según la CEPAL cabe preguntarse si la actual crisis financiera internacional es un accidente ocurrido en un sistema financiero global básicamente bien diseñado o representa una ruptura fundamental y estructural con el sistema actual, que exige un nuevo orden financiero internacional. Sea cual fuere la respuesta, en épocas de contracción los formuladores de política deberán poner más énfasis en la elaboración de políticas anti cíclicas para enfrentar las peores condiciones económicas y financieras.

\section{Efectos de la crisis en América Latina y el Caribe}

Según la CEPAL se prevé que el impacto de la crisis económica y financiera mundial en las economías de América Latina y el Caribe se canalizará por cinco vías:

- Contagio financiero y endeudamiento externo.

- Inversión extranjera.

- Demanda externa.

- Remesas de los trabajadores y

- Cambios de los precios relativos (sobre todo de los productos básicos).

En consecuencia los efectos de la crisis se propagarán tanto a nivel macroeconómico como microeconómico y los grupos sociales más vulnerables serán los más afectados. Se espera un aumento de la pobreza, sobre todo debido al incremento de los precios de los alimentos y la energía y al deterioro de las condiciones del mercado laboral.

Tras la quiebra de Lehman Brothers, la crisis tuvo un impacto aún mayor en los

mercados financieros de América Latina y el Caribe. En los últimos cuatro meses de 2008, la entrada de inversiones de cartera a la región experimentó una desaceleración para después comenzar a disminuir. 


\section{Pensamiento Crítico Vol. I8. Nº}

Asimismo, los mercados bursátiles se desplomaron y las monedas se depreciaron drásticamente, en parte como resultado de posiciones especulativas previas basadas en expectativas de apreciación de las monedas latinoamericanas. Las empresas con deuda en moneda extranjera sufrieron un impacto negativo en sus balances a raíz de la devaluación de varias monedas de la región.

A diferencia de otras crisis, en esta ocasión el sector privado parece haber sido el más expuesto a la volatilidad del tipo de cambio en muchos países. Además, el costo del endeudamiento externo se disparó, sobre todo para las empresas, pero también para los deudores soberanos.

Pese a que la actividad financiera de la región no ha estado expuesta a activos "tóxicos", los problemas del mercado interbancario internacional y el impacto de la restricción del crédito externo en los mercados de crédito locales traspasaron a la región parte de la confusión reinante en los mercados financieros de los países desarrollados. Sin embargo, la dimensión de este impacto aún no se conoce a cabalidad.

Motivo de especial preocupación son las condiciones de acceso al crédito de algunas grandes empresas locales que suelen buscar financiamiento en los mercados internacionales. Teniendo en cuenta la restricción del crédito y su encarecimiento en los mercados financieros globales, fue más difícil cumplir con los requisitos para acceder a un préstamo.

La reducida disponibilidad del financiamiento externo obligó a las empresas privadas más grandes a recurrir a los mercados internos, que probablemente tendrán problemas de liquidez. Este hecho, sumado al aumento de la incertidumbre, pondrá en aprietos a las pequeñas y medianas empresas que deseen acceder a recursos financieros.

La restricción de las condiciones financieras internacionales también tendrá un efecto negativo en la entrada de inversión extranjera directa, que en los últimos años es una importante fuente de financiamiento para algunos países.

La recesión en los mercados desarrollados y la significativa desaceleración en las economías emergentes reducirán la demanda de exportaciones de la región. La 


\section{Raimundo Renaun Pacheco Mexzon}

posición de los Estados Unidos como importador de productos de América Latina y el Caribe ha variado considerablemente según el país.

Las importaciones de los Estados Unidos provenientes de los países andinos y el MERCOSUR (en su mayoría productos básicos) aumentaron conjuntamente con los precios de estos productos desde mediados de 2007 hasta el tercer trimestre de 2008, cuando comenzaron a estabilizarse, para restringirse actualmente.

Las importaciones de China desde América Latina y el Caribe, que provienen en su mayoría de los países andinos y el MERCOSUR, siguieron una trayectoria similar. Este país asiático también enfrenta una caída de la demanda de sus productos manufacturados por parte de los mercados desarrollados, lo que socavará su vitalidad económica y por ende su demanda a futuro de los productos básicos que exporta la región.

El debilitado mercado laboral de las economías desarrolladas está teniendo un efecto negativo en las remesas que los trabajadores emigrantes envían a sus familias en sus países de origen. Las remesas han sido una importantísima fuente de ingresos externos para América Latina y el Caribe, ya que ayudan a mejorar el bienestar de las familias de bajos ingresos.

La caída de los precios de los productos básicos tras la desaceleración del crecimiento mundial se está manifestando en un deterioro de los términos de intercambio de la región en su conjunto, aunque con diversos efectos en los distintos países.

Si bien se registró un aumento generalizado del precio de estos productos, el incremento fue especialmente llamativo en los casos del petróleo, de algunos metales, como el oro, la plata, el cobre, y de ciertos alimentos, como la soja, el maíz y el trigo. Sin embargo en la actualidad se aprecia un gran deterioro especialmente en metales.

El índice de la mayoría de las exportaciones de la región llegó a su punto máximo entre junio y agosto de 2008 y desde entonces se observaron fuertes descensos, que anunciaron la nueva fase de recesión y deflación de la crisis internacional. 


\section{Pensamiento Crítico Vol. I8. Nº}

Los productos básicos constituyen una proporción considerable de la canasta de exportaciones de muchos países de América Latina y el Caribe y en algunos también son una importante fuente de ingresos públicos. Por lo tanto, se espera que el descenso o la volatilidad de los precios de los productos básicos provoquen la paralización de uno de los recientes motores del crecimiento regional. En Chile y el Perú, ambos exportadores de metales, se aprecia que los términos de intercambio han sufrido un deterioro.

\section{Crisis y Valor Económico Agregado de la Empresa}

Desde el año 2008 en que se inicia la crisis financiera en Estados Unidos de Norte América y se expande la misma afectando a otros países desarrollados se ha convertido en una crisis económico - financiera mundial que amenaza la esfera social y empresarial del mundo.

En este contexto las empresas vienen desarrollando sus operaciones por lo tanto su creación de valor económico en el mercado también se verá afectado por las implicancias de la crisis en los resultados futuros que estas puedan generar.

Para comprobar esta hipótesis definiremos que el Valor Económico Agregado de la empresa es igual a la utilidad operativa después de impuestos menos el producto del costo de capital promedio ponderado con la inversión neta en activos operativos.

La fórmula para calcular este valor será:

$$
E V A=N O P A T-W A C C * N I O A
$$

\section{Donde:}

EVA = Valor Económico Agregado de la empresa

NOPAT $=$ Utilidad operativa neta de impuestos

WACC $=$ Costo de capital promedio ponderado

NIOA = Inversión neta en activos operativos 


\section{Raimundo Renaun Pacheco Mexzon}

\section{EL VALOR ECONÓMICO AGREGADO}

Es el importe que queda una vez que se han deducido de los ingresos la totalidad de los gastos incurridos incluyendo el costo de oportunidad del capital. Sus siglas EVA provienen de su nombre en inglés.

\section{EVA}
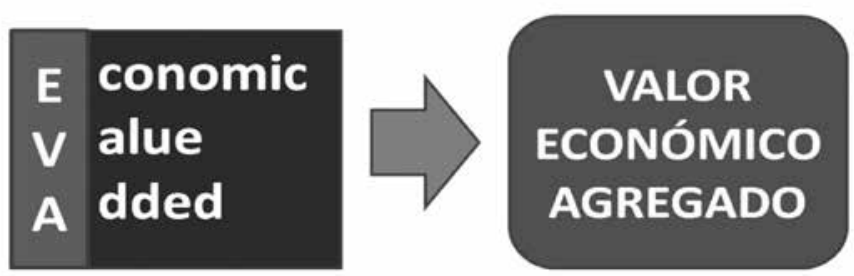

\section{El NOPAT}

Es la utilidad operativa neta después de impuestos. Se calcula partiendo del Estado de Ganancias y Pérdidas. Sus siglas NOPAT provienen de su nombre en inglés.

\section{NOPAT}

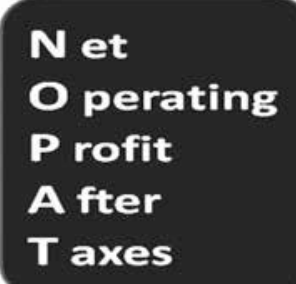

Ganancia

Operativa

Neta

Después de

Impuestos 


\section{Pensamiento Crítico Vol. 18. No I}

\section{EL WACC}

Es el costo de capital promedio ponderado. Se calcula partiendo del Balance General. Sus siglas WACC provienen de su nombre en inglés.

\section{WACC}

W eighted

A verage

Cost of

Capital
Costo de

Capital

Promedio

Ponderado

\section{EL NIOA}

Es la inversión neta en activos operativos. Se calcula partiendo del Balance General. Sus siglas NIOA provienen de su nombre en inglés.

\section{NIOA}

\section{$\mathbf{N}$ et}

I nvestment

O perating

A ssets

\section{Inversión}

Neta en

Activos

operativos 


\section{Raimundo Renaun Pacheco Mexzon}

\section{Cálculo del EVA de una Empresa}

Una empresa que está operando en nuestro país y que actúa en el mercado internacional, presenta hoy sus estados financieros auditados de Ganancias y Pérdidas y Balance General tal conforme se indica a continuación:

ESTADO DE GANANCIAS Y PÉRDIDAS (Miles de US\$)

\begin{tabular}{|c|c|}
\hline Ventas Netas & 2,600 \\
\hline Costo de ventas & 1,400 \\
\hline Utilidad Bruta & 1,200 \\
\hline Gastos operativos & 650 \\
\hline Utilidad Operativa & 550 \\
\hline Gastos financieros & 200 \\
\hline $\begin{array}{l}\text { Utilidad antes de Impuestos } \\
\end{array}$ & 350 \\
\hline Impuesto a la renta (30 \%) & 105 \\
\hline Utilidad neta después de Impuestos & 245 \\
\hline Ganancias extraordinarias & 50 \\
\hline Pérdidas extraordinarias & -30 \\
\hline Utilidad Neta & 265 \\
\hline
\end{tabular}




\section{Pensamiento Crítico Vol. 18. No I}

BALANCE GENERAL (Miles de US\$)

\begin{tabular}{|lc|}
\hline ACTIVO CORRIENTE & $\mathbf{8 3 5}$ \\
\hline Caja & 85 \\
Cuentas por cobrar & 370 \\
Existencias & 235 \\
Otros activos corrientes & 145 \\
\hline ACTIVO FIJO NETO & $\mathbf{1 , 5 5 0}$ \\
\hline ACTIVO TOTAL & $\mathbf{2 , 3 8 5}$ \\
\hline PASIVO CORRIENTE & $\mathbf{6 5 0}$ \\
\hline Proveedores & 100 \\
Otros pasivos corrientes & 250 \\
Deuda de corto plazo & 300 \\
\hline DEUDA DE MEDIANO Y LARGO PLAZO & $\mathbf{7 6 0}$ \\
\hline PATRIMONIO & $\mathbf{9 7 5}$ \\
\hline Capital social & 300 \\
Utilidades retenidas & 430 \\
Utilidades del Ejercicio & 245 \\
\hline TOTAL PASIVOS Y PATRIMONIO & $\mathbf{2 , 3 8 5}$ \\
\hline
\end{tabular}

Con esta información presentada en los estados financieros, calculamos las utilidades operativas netas después de impuestos (NOPAT) y la inversión neta en activos operativos (NIOA).

\section{Cálculo del NOPAT}

Hay dos formas de calcular las utilidades operativas netas después de impuesto:

NOPAT $=$ Ut. N. + GF(1- T) - Ut. Ext. + Per. Ext.

NOPAT $=$ Ut. Op. ${ }^{*}(1-$ T $)$

Donde:

Ut.N. = Utilidad neta 


\section{Raimundo Renaun Pacheco Mexzon}

GF $\quad=$ Gastos financieros

T= Tasa de impuestos a la renta

Ut. Ext. = Utilidad extraordinaria

Per. Ext. = Pérdida extraordinaria

Ut. Op. = Utilidad operativa

Primer cálculo:

NOPAT $=$ Ut. N. + GF(1- T) - Ut. Ext. + Per. Ext.

NOPAT $=265+200 *(1-0.30)-50+30$

$\mathrm{NOPAT}=265+140-50+30$

NOPAT $=385$

Segundo cálculo:

NOPAT $=$ Ut. Op $*(1-\mathrm{T})$

$\mathrm{NOPAT}=550 *(1-0.30)$

NOPAT $=385$

Cálculo del NIOA

Hay dos formas de calcular la inversión neta en activos operativos:

NIOA $=$ Activo Total - Proveedores - Otros Pasi. Cts.

NIOA = Patrimonio + DFCP + DFMyLP

Donde:

NIOA = Inversión neta en activos operativos

Pasi. Cts. $=$ Pasivos corrientes 


\section{Pensamiento Crítico Vol. 18. No I}

DFCP = Deuda financiera de corto plazo

DFMyLP = Deuda financiera de mediano y largo plazo

Primer cálculo

$\mathrm{NIOA}=$ Activo Total - Proveedores - Otros Pasi. Cts.

$\mathrm{NIOA}=2,385-100-250$

NIOA $=2,035$

Segundo cálculo:

$\mathrm{NIOA}=$ Patrimonio + DFCP + DFMyLP

$\mathrm{NIOA}=975+300+760$

$\mathrm{NIOA}=2,035$

\section{Cálculo del WACC}

Para el cálculo del costo de capital promedio ponderado se utiliza el siguiente modelo:

WACC $=\mathbf{P} /(\mathbf{P}+\mathrm{D}) * C \mathbf{k p}+\mathrm{D} /(\mathbf{P}+\mathrm{D}) * \mathrm{Cd}(\mathbf{1 - T})$

Donde:

$\mathbf{P}=$ Patrimonio

D = Deuda

$\mathbf{C k p}=$ Costo del capital propio

$\mathbf{C d}=$ Costo de la deuda

$\mathbf{T}=$ Tasa de impuesto a la renta 


\section{Raimundo Renaun Pacheco Mexzon}

Con la información de los estrados financieros y memorias de la empresa se tiene los siguientes datos :

$$
\begin{array}{ll}
\mathrm{P} /(\mathrm{P}+\mathrm{D}) & =41 \% \\
\mathrm{D} /(\mathrm{P}+\mathrm{D}) & =59 \% \\
\mathrm{Cd} & =8 \% \\
\mathrm{~T} & =30 \% \\
\mathrm{Ckp} & =13 \%
\end{array}
$$

Al reemplazar los datos en la fórmula se obtiene:

$\mathrm{WACC}=\mathrm{P} /(\mathrm{P}+\mathrm{D}) * \mathrm{Ckp}+\mathrm{D} /(\mathrm{P}+\mathrm{D}) * \mathrm{Cd}(1-\mathrm{T})$

WACC $=0.41 * 0.13+0.59 * 0.08 *(1-0.30)$

WACC $=0.1005=10.05 \%$

De esta manera el valor económico agregado sería:

$$
\text { EVA }=\text { NOPAT }- \text { WACC } * \text { NIOA }
$$

Como se conoce lo siguiente:

NOPAT $=385$

$\mathrm{NIOA}=2,035$

WACC $=10.05 \%$

Entonces:

$E V A=385-10.05 \% * 2,035$

$E V A=385-204.52$

$$
\text { EVA }=180.48
$$




\section{Pensamiento Crítico Vol. I8. Nº}

Este resultado nos está indicando que la empresa está creando valor económico por 180 millones 480 mil dólares.

Supongamos que por efecto de la crisis el próximo año las utilidades operativas que son de 550 millones llegaran solamente a 500 millones sólo por menores ingresos por caída de las ventas, entonces el NOPAT sería 350. Con este resultado el nuevo EVA sería de 145 millones 480 mil dólares. Es decir se estaría creando menos valor económico por 35 millones de dólares.

La situación se agudiza si por efecto de la crisis además de disminuir las ventas también crecen los costos operativos y por lo tanto disminuyen más las utilidades netas operativas de la empresa.

Si por efecto de la crisis también se incrementan los costos financieros y por lo tanto el WACC los resultados pueden ser catastróficos porque si el EVA sale negativo la empresa no estaría creando valor económico sino destruyendo valor.

Para graficar lo mencionado anteriormente presentemos el estado de Ganancias y Pérdidas afectado por menores ingresos por ventas y mayores costos operativos. Suponiendo que el efecto en ambos rubros es sólo del $2 \%$, tenemos lo siguiente:

ESTADO DE GANANCIAS Y PÉRDIDAS (Miles de US\$)

\begin{tabular}{|c|c|}
\hline Ventas Netas & 2,548 \\
\hline Costo de ventas & 1,428 \\
\hline Utilidad Bruta & 1,120 \\
\hline Gastos operativos & 663 \\
\hline Utilidad Operativa & 457 \\
\hline Gastos financieros & 200 \\
\hline $\begin{array}{r}\text { Utilidad antes de Impuestos } \\
\end{array}$ & 257 \\
\hline Impuesto a la renta (30\%) & 77 \\
\hline Utilidad neta después de Impuestos & 180 \\
\hline Ganancias extraordinarias & 50 \\
\hline Pérdidas extraordinarias & -30 \\
\hline Utilidad Neta & 200 \\
\hline
\end{tabular}




\section{Raimundo Renaun Pacheco Mexzon}

Con este nuevo resultado el NOPAT sería:

NOPAT $=$ Ut. Op.*(1-T)

NOPAT $=457 *(1-0.30)$

\section{NOPAT $=319.9$}

Manteniendo la misma inversión neta en activos operativos $(2,035)$ y el WACC incrementado también en $2 \%(10.05 \%$ x $1.02=10.25 \%)$ el nuevo EVA sería:

$\mathrm{EVA}=319.9-10.25 \% * 2,035$

$E V A=319.9-208.61$

EVA $=111.29$

Con este resultado se estaría creando menor valor económico por 69 millones 190 mil dólares.

\section{CONCLUSIONES}

1. La crisis financiera que se inició el año 2008 en Estados Unidos de Norte América, se ha transformado en una crisis económica de proporción mundial y después de cinco años está amenazando en convertirse en una crisis social y empresarial con consecuencias impredecibles.

2. En este marco las empresas de todos los países incluyendo las nuestras están creando menor valor económico situación que puede convertirse en catastrófica si el EVA resultara negativo. En este caso en vez de crear valor económico la empresa estaría destruyendo valor económico.

3. En el ejemplo desarrollado encontramos que de mantenerse la crisis la empresa crearía menos valor económico pudiendo destruir valor si el EVA sale negativo. El resumen de este resultado se muestra a continuación. 


\section{Pensamiento Crítico Vol. 18. No I}

VALOR ECONÓMICO AGREGADO (EVA)

EVA de la empresa sin crisis mundial

EVA de la empresa con crisis mundial

Monor Valor Económico Agregado
MILLONES DE DÓLARES

180.48

111.29

69.19

\section{RECOMENDACIONES}

1. Sin desconocer que en cada país las empresas deben solucionar sus propios problemas, todos los países del mundo especialmente los más desarrollados deben enfrentar en conjunto la solución de esta crisis apoyados por todos los organismos internacionales.

2. Las gerencias de las empresas deben estar atentas a enfrentar las consecuencias de esta crisis tomando medidas para conservar o mejorar la creación de valor económico de la empresa por lo tanto deben periódicamente hacer el ejerció de revisar sus políticas para mejorar sus utilidades operativas, reducir costos y realizar inversiones productivas.

\section{BIBLIOGRAFÍA}

CEPAL, "La actual crisis financiera internacional y sus efectos en América Latina y el Caribe" LC/L.2999. Enero $2009 . \quad$ Pag. 3-12

Forssyth, Juan Alberto, "Finanzas Empresariales: rentabilidad y valor" Ediciones FJA 2006. Capítulo 2 pág. $62-68$

Diario Gestión, "Evolución y dimensión de la crisis internacional" OPINIÓN pag. 23 12 de noviembre del 2012.

Oriol Amat, "EVA Valor Económico Agregado. Un nuevo enfoque para optimizar la gestión empresarial, motivar a los empleados y crear valor" norma pag. 35-86 2002.

Pacheco Mexzon, Raimundo Renaun, "EL EVA Y LA CREACIÓN DE VALOR DE LAS EMPRESAS PERUANAS: PERIODO 1999 - 2003” Tesis para optar grado de Doctor en Economía. pág. 7-13. 